Pacific

Journal of

Mathematics

QUANTUM AUTOMORPHISM GROUPS OF SMALL METRIC SPACES

TEODOR BANICA 


\title{
QUANTUM AUTOMORPHISM GROUPS OF SMALL METRIC SPACES
}

\author{
TEODOR BANICA
}

\begin{abstract}
To any finite metric space $X$ we associate the universal Hopf $\mathbb{C}^{*}$-algebra $H$ coacting on $X$. We prove that spaces $X$ having at most 7 points fall into one of the following classes: (1) the coaction of $H$ is not transitive; (2) $H$ is the algebra of functions on the automorphism group of $X$; (3) $X$ is a simplex and $H$ corresponds to a Temperley-Lieb algebra; (4) $X$ is a product of simplices and $\boldsymbol{H}$ corresponds to a Fuss-Catalan algebra.
\end{abstract}

\section{Introduction}

Let $X$ be a finite set. Consider faithful coactions $v: \mathbb{C}(X) \rightarrow \mathbb{C}(X) \otimes H$ of Hopf $\mathbb{C}^{*}$-algebras $H$ on $X$. If $H$ is commutative, it has to be of the form $\mathbb{C}(G)$, with $G$ a finite group of permutations of $X$. In the general case, a group dual example shows that such an $H$ can be noncommutative and infinite-dimensional. The universal Hopf $\mathbb{C}^{*}$-algebra $H(X)$ coacting on $X$ is constructed by Wang [1998].

If $X$ has 1,2 or 3 points then $H(X)$ is the algebra of functions on the permutation group of $X$. If $X$ has more than 4 points $H(X)$ is noncommutative and infinitedimensional. The fusion rules for its irreducible corepresentations are the same as fusion rules for irreducible representations of $\mathrm{SO}(3)$, independently of \#X.

It is a natural question to ask whether such uniformisation results still hold when adding some extra structure to $X$. The case of coactions on certain fibrations $X \rightarrow Y$ is studied in [Banica 2002b]. A universal Hopf $\mathbb{C}^{*}$-algebra $H(X \rightarrow Y)$ can be constructed and, once again, fusion rules for its corepresentations are given by the combinatorics of the Fibonacci graph, independently of the initial data.

At the level of planar algebras, $H(X)$ corresponds to the Temperley-Lieb algebra and $H(X \rightarrow Y)$ corresponds to the Fuss-Catalan algebra of Bisch and Jones [1997].

In this paper we study the more general situation when we are given a metric $d$ on $X$. A universal Hopf $\mathbb{C}^{*}$-algebra $H(X, d)$ can be constructed in this case as well. In many cases $H(X, d)$ collapses to the algebra of functions on the usual automorphism group $G(X, d)$. In other cases the coaction of $H(X, d)$ is not transitive, meaning that there exists a nonconstant function $f \in \mathbb{C}(X)$ such that $v(f)=f \otimes 1$.

MSC2000: primary 16W30; secondary 46L37, 81R50. 
The most interesting case is when $H(X, d)$ is not commutative and its coaction is transitive. For instance, if $X$ is a simplex, meaning that all distances between points are equal, $H(X, d)$ is the above $H(X)$. The algebra $H(X \rightarrow Y)$ is as well of the form $H(X, d)$, for a suitable choice of the distance $d$.

We prove that for metric spaces $(X, d)$ having at most 7 points the algebras $H(X, d)$ fall into one of four classes: commutative, nontransitive, corresponding to the Temperley-Lieb algebra, corresponding to the Fuss-Catalan algebra.

This is inspired in recent work of Bisch and Jones [2000; 2003], where these authors prove the following result. Let $P$ be a spherical $\mathbb{C}^{*}$-planar algebra satisfying the following conditions.

(1) $\operatorname{dim} P_{1}=1$ and $P$ is generated by a 2-box $b \in P_{2}$.

(2) $\operatorname{dim} P_{2}=3$.

(3) $\operatorname{dim} P_{3} \leq 13$.

Then

(4) $P$ is a Temperley-Lieb or a Fuss-Catalan algebra, or is the algebra associated to $\mathbb{Z}_{3}$, or is another algebra, associated to $\mathbb{Z}_{2}$ and $D_{5}$.

This result can be reformulated as follows (compare [Woronowicz 1988; Banica 2002a]). Let $P$ be a spherical $\mathbb{C}^{*}$-planar algebra satisfying the following conditions.

(1') $\operatorname{dim} P_{1}=1$ (transitivity) and $P$ is generated by a 2-box $b \in P_{2}$ (universality).

(2') $P$ embeds into the depth-1 planar algebra associated in [Jones 2000] to the inclusion $\mathbb{C} \subset \mathbb{C}(X)$ and $b$ corresponds in this way to a distance matrix (tannakian conditions).

(3') $\# X \leq 7$.

Then

(4') $P$ is a Temperley-Lieb or a Fuss-Catalan algebra, or there is a 4-box in $P$ which corresponds to the flip automorphism of $X \times X$ (commutativity).

We see that the general conditions (1) and $\left(1^{\prime}\right)$ are the same and that the key conditions (2) and (2') are of different nature. The conclusions (4) and (4') look a bit similar just because the auxiliary conditions (3) and ( $\left.3^{\prime}\right)$ are too strong.

The methods we use for the proof are quite different from those of Bisch and Jones. The fact that the 2-box here is given by an explicit operator brings lots of simplifications with respect to the much more general situation considered in [Bisch and Jones 2000; 2003]. As an example, Landau's exchange relation [2002], one of the main tools in classifying planar algebras, says for a 2-box $p$ that the 
image of the corresponding projection $P \in \mathscr{L}(\mathbb{C}(X))$ must be closed under multiplication, and this is very easy to check.

In fact the proof of the result above just uses our previous work on the FussCatalan algebra [2002b] plus some elementary matrix computations. It is also possible to apply the powerful results of Bisch and Jones to the Hopf $\mathbb{C}^{*}$-algebras $H(X, d)$. After making all necessary identifications, the problem is to check dimension conditions on the planar algebra of $H(X, d)$. The geometric formulation of these conditions requires some combinatorial work, and this is exactly the kind of work we do in this paper.

We don't know what happens for $\# X \geq 8$. Some exceptional examples are expected to arise, for instance in connection with complex Hadamard matrices and Krishnan-Sunder biunitary permutation matrices. Associated to such matrices are quite exotic lattice models and commuting squares, which can be described by coactions [Jones and Sunder 1997; Bhattacharyya 2002; Banica 2000]. In fact, these coactions are the second motivation for the study of the algebras $H(X, d)$. So far the universal Hopf algebras $H(X)$ haven't been useful for getting new results on vertex models, but one can hope that the Hopf algebras $H(X, d)$, which are somehow of "second level", could produce better results.

From the point of view explained by Bisch and Jones [2000], the simplest planar algebras are those generated by a 2-box, and among them, simplest are those having small dimension. There is a similar hierarchy for Hopf $\mathbb{C}^{*}$-algebras:

(I) By Woronowicz's result [1998] each unital Hopf $\mathbb{C}^{*}$-algebra is an inductive limit of pairs $(H, v)$ as in [Woronowicz 1988].

(II) By replacing $v$ with ad $(v \oplus 1)$ we may assume that $v: A \rightarrow A \otimes H$ is a coaction on a finite-dimensional $\mathbb{C}^{*}$-algebra.

(III) We say that $(H, v)$ has length $k$ if it is isomorphic to the universal pair $(K, w)$ satisfying $\operatorname{Hom}\left(1, v^{\otimes k}\right) \subset \operatorname{Hom}\left(1, w^{\otimes k}\right)$. (This means that the associated planar algebra has planar depth $k$ in the sense of [Landau and Sunder 2002].) By analogy with discrete groups, each pair $(H, v)$ is a projective limit of length $k$ pairs.

(IV) The simplest situation is when $k=2$. Here $(H, v)$ is presented by the relations $d v=v d$, where $d$ is a generator of $\operatorname{Hom}\left(1, v^{\otimes 2}\right) \simeq \operatorname{End} v$ (the "2-box").

(V) The simplest case is when $A$ is abelian and $d$ is a metric on its spectrum $X$. Here $H=H(X, d)$.

Generalisations of the algebras $H(X, d)$ can be found by going up through this hierarchy. On the other side, when trying to generalise a metric space, the first thought goes to Connes' notion of a spectral triple ([Connes 1996]). There are 
many problems here, the main one being probably to find a good hierarchy of spectral triples, reflecting somehow the corepresentation theory complexity of the associated Hopf $\mathbb{C}^{*}$-algebra (if any). See [Paschke and Sitarz 1998; Rieffel 2004] for more comments on this subject.

The paper is organised as follows. Section 1 is preliminary, with material from [Wang 1998; Banica 2002b]. In Section 2 we establish some basic facts about coactions on finite metric spaces. In Section 3 we find some geometric rules for detecting commutativity or nontransitivity of $H(X, d)$, the main tool being diagonalisation of distance matrices. In Section 4 we assume that $\# X \leq 7$ and we prove the main result.

\section{Coactions on finite sets}

Let $X$ be a finite set. We denote by $\mathbb{C}(X)$ the algebra of complex functions on $X$. We use the canonical involution and trace of $\mathbb{C}(X)$, given by

$$
f^{*}(i)=\overline{f(i)}, \quad \operatorname{tr}(f)=\sum_{i} f(i) .
$$

For $K$ a set we denote by $M_{X}(K)$ the set of functions from $X \times X$ to $K$. Such a function can be written as a matrix $\left(v_{i j}\right)$ with indices $i, j$ in $X$ and entries $v_{i j}$ in $K$.

The group of permutations of $X$ is denoted by $\mathscr{S}_{X}$.

Let $H$ be the Hopf $\mathbb{C}^{*}$-algebra associated to a compact quantum group of Kac type. That is, $H$ is a unital $\mathbb{C}^{*}$-algebra together with linear maps

$$
\Delta: H \rightarrow H \otimes H, \quad \varepsilon: H \rightarrow \mathbb{C}, \quad S: H \rightarrow H,
$$

called comultiplication, counit and antipode, and subject to the following axioms. The maps $\Delta$ and $\varepsilon$ are morphisms of $\mathbb{C}^{*}$-algebras satisfying the conditions

$$
(\mathrm{id} \otimes \Delta) \Delta=(\Delta \otimes \mathrm{id}) \Delta, \quad(\mathrm{id} \otimes \varepsilon) \Delta=(\varepsilon \otimes \mathrm{id}) \Delta=\mathrm{id},
$$

and the antipode is an antimultiplicative involutive map satisfying

$$
m(\mathrm{id} \otimes S) \Delta=m(S \otimes \mathrm{id}) \Delta=\mathrm{id}, \quad S^{2}=\mathrm{id} .
$$

By [Woronowicz 1987] the Hopf $\mathbb{C}^{*}$-algebra $H$ has a Haar functional and the dense $*$-subalgebra $H_{s}$ of "smooth functions on the quantum group" is a cosemisimple Hopf $*$-algebra.

Definition 1.1. A coaction of $H$ on $X$ is a morphism of complex $*$-algebras

$$
v: \mathbb{C}(X) \rightarrow \mathbb{C}(X) \otimes H_{S}
$$

satisfying the coassociativity condition $(v \otimes \mathrm{id}) v=(\mathrm{id} \otimes \Delta) v$, the counitality condition $(\mathrm{id} \otimes \varepsilon) v=\mathrm{id}$ and the trace-preservation condition $(\operatorname{tr} \otimes \mathrm{id}) v=\operatorname{tr}(\cdot) 1$. 
Consider the basis of $\mathbb{C}(X)$ formed by the Dirac masses $\delta_{i}$ with $i \in X$. The coaction $v$ is given by the sum

$$
v\left(\delta_{i}\right)=\sum_{j} \delta_{j} \otimes v_{j i} .
$$

The elements $v_{i j}$ are called coefficients of the coaction. A coaction is called faithful if its coefficients generate the $*$-algebra $H$. In general we have a factorisation

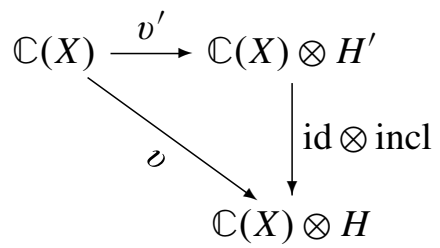

where $H^{\prime} \subset H$ is the $*$-algebra generated by the coefficients of $v$. From the axioms for $v$ we get that $H^{\prime}$ is stable under comultiplication, so $v^{\prime}$ is a coaction as well. Thus any coaction factorises through a faithful coaction.

As a first example, consider the Hopf $\mathbb{C}^{*}$-algebra $\mathbb{C}(G)$ associated to a finite group $G$. Here the comultiplication, counit and antipode are obtained by applying the $\mathbb{C}$ functor to the multiplication, unit and inverse map of $G$ :

$$
\Delta\left(\delta_{g}\right)=\sum_{h k=g} \delta_{h} \otimes \delta_{k}, \quad \varepsilon(f)=f(1), \quad S\left(\delta_{g}\right)=\delta_{g^{-1}}
$$

If $(x, g) \mapsto g x$ is an action of $G$ on $X$, by applying the the $\mathbb{C}$ functor we get a map from $\mathbb{C}(X)$ to $\mathbb{C}(X \times G) \simeq \mathbb{C}(X) \otimes \mathbb{C}(G)$, which is a coaction:

$$
v\left(\delta_{i}\right)=\sum_{g} \delta_{g i} \otimes \delta_{g}=\sum_{j} \delta_{j} \otimes \sum_{g i=j} \delta_{g} .
$$

This shows that $v$ is faithful if and only if the action map $G \rightarrow \mathscr{Y}_{X}$ is injective. Thus any subgroup of the symmetric group $\mathscr{S}_{X}$ produces a faithful coaction on $X$.

Note that the trace-preservation condition says that the action of $G$ must preserve the counting measure on $X$, and this is always true. In general this condition is not satisfied, and in case it does not hold unwanted decomposition appears in the corepresentation theory of $H$ [Wang 1998]. In topological terms, the tracepreservation condition is needed in order to avoid "floating circles" in the corresponding planar algebra. See [Jones 2000; Banica 2002a].

Note also that the Kac algebra assumption $S^{2}=$ id is satisfied for $H=\mathbb{C}(G)$. In general, this assumption cannot be dropped, in the sense that for any Hopf $\mathbb{C}^{*}$ algebra coacting faithfully on $\mathbb{C}(X)$ the square of the antipode must be the identity. 
The axioms for $v$ can be translated in terms of its coefficients $v_{i j}$. First, the fact that $v$ is coassociative and counital translates into two "comultiplicative" formulae,

$$
\Delta\left(v_{i j}\right)=\sum_{k} v_{i k} \otimes v_{k j} \quad \text { and } \quad \varepsilon\left(v_{i j}\right)=\delta_{i j} .
$$

These conditions say that $v$ is a corepresentation of $H$ on the linear space $\mathbb{C}(X)$ :

$$
(\mathrm{id} \otimes \Delta) v=v_{12} v_{13}, \quad(\mathrm{id} \otimes \varepsilon) v=1 .
$$

For the other axioms, note first that the structure of $\mathbb{C}(X)$ is given by the formulae

$$
\delta_{i}^{2}=\delta_{i}, \quad \delta_{i} \delta_{I}=0, \quad 1=\sum_{l} \delta_{l}, \quad \delta_{i}^{*}=\delta_{i}, \quad \operatorname{tr}\left(\delta_{i}\right)=1
$$

valid for any $i$ and any $I \neq i$. Thus the fact that $v$ is a multiplicative, unital, involutive and trace-preserving translates into the following "multiplicative" formulae.

$$
v_{j i}^{2}=v_{j i}, \quad v_{j i} v_{j I}=0, \quad \sum_{l} v_{j l}=1, \quad v_{j i}^{*}=v_{j i}, \quad \sum_{j} v_{j i}=1 .
$$

The first four formulae say that each row of $v$ is a partition of the unity of $H$ with self-adjoint projections. In particular the matrix $v^{*} v$ is diagonal. The diagonal entries of $v^{*} v$ being the sums in the fifth formula, this formula says that $v$ satisfies $v^{*} v=1$. Since $v$ is a corepresentation, it follows that $v$ is unitary and that the antipode is given by the "third comultiplicative formula",

$$
S\left(v_{i j}\right)=v_{j i} .
$$

By applying $S$ to the second multiplicative formula we conclude that the columns of $v$ are also partitions of unity with self-adjoint projections.

Summing up, in order for the map $v$ to be a coaction the matrix $v$ must be a corepresentation, as well as a magic biunitary in the following sense.

Definition 1.2. A matrix $v \in M_{X}(K)$ over a complex $*$-algebra $K$ is called a magic biunitary if its lines and columns are partitions of the unity of $K$ with projections. (This is reminiscent of the definition of a magic square in combinatorics.)

Let $Z_{i}$ be a finite sets and let

$$
v_{i}: \mathbb{C}\left(Z_{i}\right) \rightarrow \mathbb{C}\left(Z_{i}\right) \otimes H_{i}
$$

be coactions. Consider the corresponding magic biunitary corepresentations $v_{i}$. Then the matrix

$$
\bigoplus v_{i}=\left(\begin{array}{cccc}
v_{1} & 0 & \cdots & 0 \\
0 & v_{2} & \cdots & 0 \\
\vdots & \vdots & \ddots & \vdots \\
0 & 0 & \cdots & v_{n}
\end{array}\right)
$$


is a magic biunitary corepresentation of the free product $* H_{i}$, so we get a coaction of $* H_{i}$ on the disjoint union $\sqcup Z_{i}$.

$$
\bigoplus v_{i}: \mathbb{C}\left(\bigsqcup Z_{i}\right) \rightarrow \mathbb{C}\left(\bigsqcup Z_{i}\right) \otimes\left(* H_{i}\right) .
$$

This coaction is faithful if and only if all $v_{i}$ are faithful.

As a first example, let $n_{i}$ be positive integers. For each $i$ the canonical action of the cyclic group $\mathbb{Z}_{n_{i}}$ on itself produces a faithful coaction - the comultiplication

$$
\Delta_{i}: \mathbb{C}\left(\mathbb{Z}_{n_{i}}\right) \rightarrow \mathbb{C}\left(\mathbb{Z}_{n_{i}}\right) \otimes \mathbb{C}\left(\mathbb{Z}_{n_{i}}\right) .
$$

The free product $* \mathbb{C}\left(\mathbb{Z}_{n_{i}}\right)$ can be computed by using the Fourier transform over cyclic groups and the distributivity of the free product of Hopf $*$-algebras with respect to the $\mathbb{C}^{*}$ functor for discrete groups [Wang 1995]:

$$
* \mathbb{C}\left(\mathbb{Z}_{n_{i}}\right) \simeq * \mathbb{C}^{*}\left(\mathbb{Z}_{n_{i}}\right) \simeq \mathbb{C}^{*}\left(* \mathbb{Z}_{n_{i}}\right) .
$$

We get in this way a faithful coaction

$$
\oplus \Delta_{i}: \mathbb{C}\left(\sqcup \mathbb{Z}_{n_{i}}\right) \rightarrow \mathbb{C}\left(\sqcup \mathbb{Z}_{n_{i}}\right) \otimes \mathbb{C}^{*}\left(* \mathbb{Z}_{n_{i}}\right) .
$$

In particular for any $n \geq 4$ we can take $n_{1}=2$ and $n_{2}=n-2$ and we get a faithful coaction of $\mathbb{C}^{*}\left(\mathbb{Z}_{2} * \mathbb{Z}_{n-2}\right)$ on the set with $n$ points. Thus $H$ can be infinitedimensional. Together with the above remarks on the square of the antipode and on the trace-preservation condition, this justifies the formalism in Definition 1.2.

The universal Hopf $\mathbb{C}^{*}$-algebra coacting on $X$ is constructed in [Wang 1998].

Definition 1.3. The universal $\mathbb{C}^{*}$-algebra $H(X)$ is defined with generators $v_{i j}$ with $i, j \in X$ and with the relations making $v$ a magic biunitary.

The comultiplication, counit of antipode of $H(X)$ are constructed by using its universal property. Thus $H(X)$ is a Hopf $\mathbb{C}^{*}$-algebra coacting on $X$. For any coaction $v: \mathbb{C}(X) \rightarrow \mathbb{C}(X) \otimes H$ we have the factorisation diagram

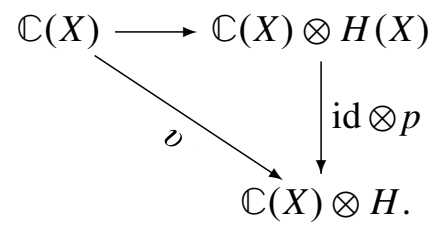

In particular we have a morphism $p_{\text {com }}: H(X) \rightarrow \mathbb{C}(G(X))$ onto the algebra of functions on the usual symmetric group $G(X)$. For sets having 1,2 or 3 points this map $p_{\text {com }}$ is an isomorphism. If $X$ has at least 4 points the free product example above shows that $H(X)$ has quotients of the form $\mathbb{C}^{*}(\Gamma)$ with $\Gamma$ infinite discrete group. In particular $H(X)$ must be noncommutative and infinite-dimensional, and $p_{\text {com }}$ cannot be an isomorphism. See [Wang 1998]. 
Some useful information about $H(X)$ can be obtained by using corepresentation theory techniques. Woronowicz [1988] finds a general Tannaka-Krein type duality and applies it to the algebra $H=\mathbb{C}(\operatorname{SU}(N))_{q}$ with $q>0$. The idea is that

(1) generators and relations defining $H$ translate into a presentation result of the associated tensor category, and

(2) these latter equations turn out to have a diagrammatic solution, and using diagrams one can compute various corepresentation theory invariants of $H$.

The same method works for $H=H(X)$. Consider the multiplication and the unit operator of the algebra $\mathbb{C}(X)$.

$$
m: \mathbb{C}(X) \otimes \mathbb{C}(X) \rightarrow \mathbb{C}(X), \quad u: \mathbb{C} \rightarrow \mathbb{C}(X) .
$$

These are arrows in the tensor $\mathbb{C}^{*}$-category whose objects are tensor powers of $\mathbb{C}(X)$, and where arrows are linear maps between such tensor powers. Let $\langle m, u\rangle$ be the tensor $\mathbb{C}^{*}$-category generated by $m$ and $u$. This is by definition the smallest tensor $\mathbb{C}^{*}$-category containing $m$ and $u$. Its objects are the tensor powers of $\mathbb{C}(X)$, and arrows are all possible linear combinations of compositions of tensor products of maps of the form $m, u, m^{*}, u^{*}$, id, where id is the identity of $\mathbb{C}(X)$.

Since $v$ is multiplicative and unital we have

$$
m \in \operatorname{Hom}\left(v^{\otimes 2}, v\right), \quad u \in \operatorname{Hom}(1, v) .
$$

Thus both $m, u$ are arrows in the category $C$ of finite-dimensional corepresentations of $H(X)$. The category $\langle m, u\rangle$ above can be regarded as a subcategory of $C$.

On the other hand both $m \in \operatorname{Hom}\left(v^{\otimes 2}, v\right)$ and $u \in \operatorname{Hom}(1, v)$ give rise to a collection of relations between coefficients $v_{i j}$, which are easily seen to be equivalent to those expressing the magic biunitary condition. At the level of categories, this means that $C$ is the completion of $\langle m, u\rangle$, meaning the smallest semisimple tensor $\mathbb{C}^{*}$-category containing $\langle m, u\rangle$.

This was step (1) in Woronowicz's method. What is left is step (2), namely the computation of $\langle m, u\rangle$ by using some combinatorial tool, such as planar diagrams. The diagrams needed here are those related to the Temperley-Lieb algebra. There are several approaches to them, beginning with those in [Jones 1985] and [Kauffman 1987]. Here we use a formalism close to the one in [Bisch and Jones 1997].

Set $\delta_{X}=\sqrt{\# X}$ and consider the category $T L^{2}\left(\delta_{X}\right)$ whose objects are positive integers and where arrows between $k$ and $l$ are linear combinations of TemperleyLieb diagrams between $2 k$ and $2 l$ points. (Such a diagram has $k+l$ noncrossing strings joining points, etc.) Here are the operations: The composition is vertical concatenation, the tensor product is horizontal concatenation, the involution is 
upside-down turning, and erasing a floating circle is the same as multiplying by $\delta_{X}$ :

$$
\mathrm{A} \circ \mathrm{B}=\mathrm{B}, \quad \mathrm{A} \otimes \mathrm{B}=\mathrm{AB}, \quad \mathrm{A}^{*}=\mathrm{V}, \quad \bigcirc=\delta_{X} .
$$

This tensor $\mathbb{C}^{*}$-category is generated by the diagrams

$$
M=\delta_{X}^{1 / 2}|\cup| \text { and } U=\delta_{X}^{-1 / 2} \cap,
$$

and a direct computation shows that $M \mapsto m$ and $U \mapsto u$ give an isomorphism between $T L^{2}\left(\delta_{X}\right)$ and $\langle m, u\rangle$. Thus the category of finite-dimensional corepresentations of $H(X)$ is isomorphic to the completion of $T L^{2}\left(\delta_{X}\right)$. See [Banica 2002b].

Let $Y, Z$ be finite sets and consider the product $X=Y \times Z$. The projection $X \rightarrow Y$ gives a unital embedding $\imath: \mathbb{C}(Y) \rightarrow \mathbb{C}(X)$, so we can consider coactions on $X$ which restrict to coactions on $Y$ :

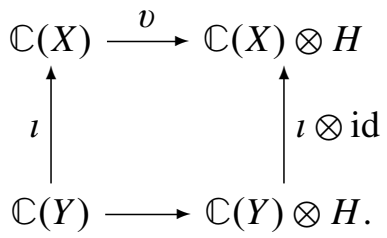

For a coaction $v$ to restrict to $\mathbb{C}(Y)$, it must send $\mathbb{C}(Y)$ into $\mathbb{C}(Y) \otimes H$.

Definition 1.4. The Hopf $\mathbb{C}^{*}$-algebra $H(X \rightarrow Y)$ is the quotient of $H(X)$ by the closed two-sided $*$-ideal generated by the relations coming from the equality $e v=v e$, where $e: \mathbb{C}(X) \rightarrow \mathbb{C}(X)$ is the linear map implemented by the projection $X \rightarrow Y$.

The Hopf $\mathbb{C}^{*}$-algebra $H(X \rightarrow Y)$ coacts on $X$ and the coaction restricts to $Y$. For any coaction on $X$ which restricts to $Y$ we have the factorisation diagram

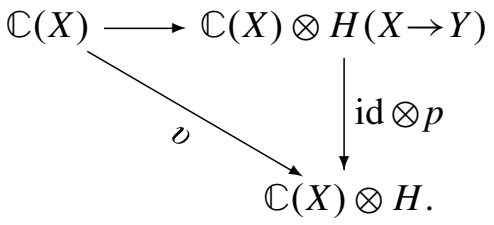

The category of corepresentations of $H(X \rightarrow Y)$ can be computed by using the same techniques as for $H(X)$, with the Fuss-Catalan diagrams of Bisch and Jones [1997].

First, the universal property of $H(X \rightarrow Y)$ translates into the fact that its category of corepresentations is the completion of the tensor $\mathbb{C}^{*}$-category generated by $m$, $u$ and $e$. Set $\delta_{Y}=\sqrt{\# Y}$ and $\delta_{Z}=\sqrt{\# Z}$ and consider the category $F C\left(\delta_{Y}, \delta_{Z}\right)$ whose objects are positive integers and where the arrows between $l$ and $k$ are linear 
combinations of Fuss-Catalan diagrams between $4 l$ points and $4 k$ points. These are by definition Temperley-Lieb diagrams with both rows of points colored

$$
y, z, z, y, y, z, z, y, \ldots,
$$

such that strings join points having the same color. Then $F C\left(\delta_{Y}, \delta_{Z}\right)$ is a tensor $\mathbb{C}^{*}$-category, with operations given by usual horizontal and vertical concatenation and upside-down turning of diagrams, plus the following rule: erasing a $y$-colored or $z$-colored circle is the same as multiplying the diagram by $\delta_{Y}$ or $\delta_{Z}$.

$$
y \text {-colored } \rightarrow \bigcirc=\delta_{Y}, \quad z \text {-colored } \rightarrow \bigcirc=\delta_{Z} .
$$

With $\delta_{X}=\delta_{Y} \delta_{Z}=\sqrt{\# X}$ this category is generated by the diagrams

$$
M=\delta_{X}^{1 / 2}\|\uplus\|, \quad U=\delta_{X}^{-1 / 2} \cap, \quad E=\delta_{Z}^{-1 / 2} \mid \begin{aligned}
& \bigcap \\
& \cap
\end{aligned},
$$

and computation shows that $M \mapsto m, U \mapsto u$ and $E \mapsto e$ give an isomorphism between $F C\left(\delta_{Y}, \delta_{Z}\right)$ and $\langle m, u, e\rangle$. Thus the category of finite-dimensional corepresentations of $H(X \rightarrow Y)$ is isomorphic to the completion of $F C\left(\delta_{Y}, \delta_{Z}\right)$. See [Banica 2002b].

Finally, let us mention that quite exotic examples, not to be discussed in this paper, can be obtained from magic biunitary matrices. Let $X, Y$ be finite sets and let $\left(u_{i j}\right)$ be a magic biunitary with $i, j \in X$ and $u_{i j} \in M_{Y}(\mathbb{C})$. The universal property of $H(X)$ gives a representation on $M_{Y}(\mathbb{C})$ sending the fundamental corepresentation $v$ to $u$ :

$$
\pi: H(X) \rightarrow M_{Y}(\mathbb{C}), \quad(\operatorname{id} \otimes \pi) v=u .
$$

By dividing $H(X)$ by a suitable ideal we get a factorisation

$$
\pi=\pi_{u} p_{u}
$$

through a minimal possible Hopf $\mathbb{C}^{*}$-algebra $H(u)$. This coacts on $\mathbb{C}(X)$ via $v_{u}=$ $\left(\mathrm{id} \otimes p_{u}\right) v:$

$$
\pi_{u}: H(u) \rightarrow M_{Y}(\mathbb{C}), \quad\left(\mathrm{id} \otimes \pi_{u}\right) v_{u}=u .
$$

For instance, if $G$ is a subgroup of $\mathscr{Y}_{Y}$ and $u$ is the magic biunitary corresponding to the action of $G$ on $Y$ then $H(u)=\mathbb{C}(G)$ and $v_{u}=u$.

More complicated examples come from lattice models and commuting squares. Thus, complex Hadamard matrices and Krishnan-Sunder biunitary permutation matrices are known to produce magic biunitaries [Jones and Sunder 1997; Bhattacharyya 2002; Banica 2000]. So far nothing seems to be known about the associated Hopf $\mathbb{C}^{*}$-algebras, besides their existence. 


\section{Coactions on finite metric spaces}

Let $(X, d)$ be a finite metric space. That is, $X$ is a finite set and $d: X \times X \rightarrow \mathbb{R}_{+}$ is symmetric, has 0 on the diagonal and positive values outside the diagonal, and its entries satisfy the triangle inequality.

Let $G$ be a group acting on $X$, and take the corresponding coaction of $\mathbb{C}(G)$ on $X$ :

$$
v: \mathbb{C}(X) \rightarrow \mathbb{C}(X) \otimes \mathbb{C}(G), \quad v\left(\delta_{i}\right)=\sum_{g} \delta_{g i} \otimes \delta_{g} .
$$

The products $d v$ and $v d$ are given by

$$
(d v)_{i j}=\sum_{g} d(i, g j) \delta_{g}, \quad(v d)_{i j}=\sum_{g} d\left(g^{-1} i, j\right) \delta_{g} .
$$

Thus the coaction of $\mathbb{C}(G)$ on $X$ preserves the metric if and only if the action of $G$ on $X$ preserves the metric. This suggests the following definition.

Definition 2.1. Let $H$ be the Hopf $\mathbb{C}^{*}$-algebra associated to a compact quantum group of Kac type. A coaction of $H$ on $X$ given by

$$
v: \mathbb{C}(X) \rightarrow \mathbb{C}(X) \otimes H, \quad v\left(\delta_{i}\right)=\sum_{j} \delta_{j} \otimes v_{j i}
$$

is said to preserve the metric if the matrix $v=\left(v_{i j}\right)$ commutes with $d=(d(i, j))$.

Let $H(X, d)$ be the quotient of $H(X)$ by the relations coming from $d v=v d$. Then $H(X, d)$ coacts on $\mathbb{C}(X)$ and preserves the metric. For any other coaction that preserves the metric we have the factorisation diagram

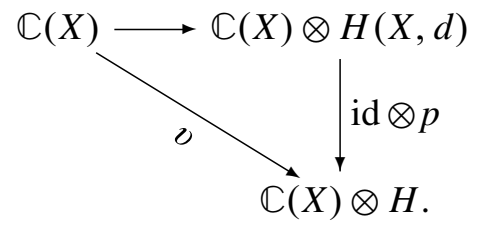

In particular we have the following factorisation of the universal coaction:

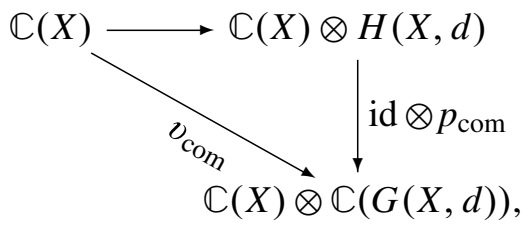

where $G(X, d)$ is the automorphism group of $(X, d)$ and $v_{\text {com }}$ is the corresponding coaction. We will see that in many cases the projection $p_{\text {com }}$ is an isomorphism.

The simplest example of a finite metric space is a finite set with the $0-1$ distance on it. More generally, we define a simplex as a finite metric space having the same distance between all pairs of disjoint points. 
Proposition 2.2. If $(X, d)$ is a simplex then any coaction on $X$ preserves the metric. We have $H(X, d)=H(X)$.

Proof. The distance on a simplex is given by the formula

$$
d=a(\mathbb{1}-1),
$$

where $a$ is the nonzero value of the distance, 1 is the identity matrix and $\mathbb{1}$ is the matrix having 1 everywhere:

$$
\mathbb{1}=\left(\begin{array}{ccc}
1 & \cdots & 1 \\
\vdots & \ddots & \vdots \\
1 & \cdots & 1
\end{array}\right)
$$

Let $v$ be the magic biunitary corepresentation corresponding to a coaction on $X$. Since right and left multiplication by $\mathbb{1}$ is making sums on rows and columns, $\mathbb{1}$ commutes with $v$. Thus $v$ commutes with $d$ as well.

The metric analogue of the free product example is as follows. Let $(Y, D)$ be a metric space and for each $i \in Y$ let $\left(Z_{i}, d_{i}\right)$ be a metric space. Assume that the condition

$$
2 D(i, j) \geq d_{i}(p, q) .
$$

is satisfied, for any $i, j \in Y$ and for any $p, q \in Z_{i}$. The disjoint union $X=\bigsqcup Z_{i}$ becomes a metric space, with distance $d$ equal to $d_{i}$ on each $Z_{i}$ and equal to $D(i, j)$ between points of $Z_{i}$ and $Z_{j}$ for $i \neq j$. It is useful to keep in mind the following picture: $(X, d)$ is made of a metric "macrospace" $(Y, D)$ having around each of its points $i$ a metric "microspace" $\left(Z_{i}, d_{i}\right)$.

Proposition 2.3. If $v_{i}: \mathbb{C}\left(Z_{i}\right) \rightarrow \mathbb{C}\left(Z_{i}\right) \otimes H_{i}$ are coactions then the coaction

$$
\bigoplus v_{i}: \mathbb{C}(X) \rightarrow \mathbb{C}(X) \otimes\left(* H_{i}\right)
$$

preserves the metric $d$ if and only if each $v_{i}$ preserves the metric $d_{i}$.

Proof. If points of $Y$ are labelled from 1 to $n$ then the distance on $X$ is

$$
d=\left(\begin{array}{cccc}
d_{1} & D(1,2) \mathbb{1} & \cdots & D(1, n) \mathbb{1} \\
D(2,1) \mathbb{1} & d_{2} & \cdots & D(2, n) \mathbb{1} \\
\vdots & \vdots & \ddots & \vdots \\
D(n, 1) \mathbb{1} & D(n, 2) \mathbb{1} & \cdots & d_{n}
\end{array}\right)
$$

where $\mathbb{1}$ denotes rectangular matrices of suitable size filled with 1's. Since multiplication by $\mathbb{1}$ is making sums on rows or columns, $\mathbb{1}$ commutes with each $v_{i}$. Thus $\bigoplus v_{i}$ commutes with $d$ is and only if each $v_{i}$ commutes with $d_{i}$. 
As an example, let $Y$ be the segment of length 1 and let $Z_{i}$ be two segments of length $\sqrt{2}$. The metric space $X$ is a square. Since $\mathbb{Z}_{2}$ acts on both segments, now diagonals of the square, it follows that $\mathbb{C}^{*}\left(\mathbb{Z}_{2} * \mathbb{Z}_{2}\right)$ coacts on the square.

More generally, let $Y$ be a simplex with nonzero distance $A$ and $Z$ be a simplex with nonzero distance $a$ and assume that $A \neq a$ and $2 A \geq a$. For any $i \in Y$ define $Z_{i}=Z$. The disjoint union in the above example is $X=Y \times Z$, with the following metric.

$$
d=\left(\begin{array}{cccc}
a(\mathbb{1}-1) & A \mathbb{1} & \cdots & A \mathbb{1} \\
A \mathbb{1} & a(\mathbb{1}-1) & \cdots & A \mathbb{1} \\
\vdots & \vdots & \ddots & \vdots \\
A \mathbb{1} & A \mathbb{1} & \cdots & a(\mathbb{1}-1)
\end{array}\right) .
$$

It is useful to keep in mind the following picture: $X$ is a space made of a "macrosimplex" $Y$, having around each of its points a copy of the "microsimplex" $Z$.

Proposition 2.4. A coaction on $X$ preserves the metric if and only if it restricts to $Y$. We have $H(X, d)=H(X \rightarrow Y)$.

Proof. We have the formula

$$
d=A \mathbb{1}+(a-A)(\# Z) e-a 1,
$$

where 1 is the identity matrix and where $e$ is the operator

$$
e=\frac{1}{\# Z}\left(\begin{array}{cccc}
\mathbb{1} & 0 & \cdots & 0 \\
0 & \mathbb{1} & \cdots & 0 \\
\vdots & \vdots & \ddots & \vdots \\
0 & 0 & \cdots & \mathbb{1}
\end{array}\right)
$$

Let $v$ be a coaction on $X$. Since commutation of $v$ with 1 and with $\mathbb{1}$ is automatic, $v$ preserves the distance if and only if it commutes with $e$. But $e$ is the projection onto the subalgebra $\mathbb{C}(Y)$ of $\mathbb{C}(X)$.

Given a finite metric space $(X, d)$ and a group $G$ acting on $X$, the fact that $G$ preserves or not the metric only depends on some equivalence class of the metric: the one that takes into account the equalities between various pairs of distances, but neglects their precise values. In other words, the induced action of $G$ on $X \times X$ must preserve the $0-1$ matrix of Kronecker symbols

$$
K(i, j, k, l)=\delta_{d(i, j), d(k, l)} .
$$

Instead of using $K$ or other purely combinatorial objects, it is convenient to use the metric and to draw pictures, but to keep in mind that the set of values of the distance function is irrelevant. We will call these values "colors" and the picture of a metric space will consist of points plus colored lines between them, with the 
convention that same color means same distance. For typographical reasons colors in this paper will be different kinds of lines, plus a "void color" that is not pictured. The void color is used in order to simplify pictures and will be typically a most frequent color, i.e., a number $a$ that maximizes the quantity

$$
\#\{(i, j) \mid d(i, j)=a\} .
$$

As an example, here is a rectangle that is not a square. The void color is here the length of the diagonals, which is different from the lengths of the sides.

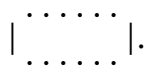

Let $(X, d)$ be a metric space with color set $C \subset \mathbb{R}_{+}$. For any color $a \in C$ we denote by $d_{a}$ the $0-1$ matrix having 1 if $d(i, j)=a$ and 0 elsewhere. The following formula will be called the color decomposition of the metric.

$$
d=\sum_{a \in C} a d_{a} .
$$

For instance the color decomposition for the rectangle having sides of lengths $a$ and $c$ and diagonals of lengths $b$ is as follows (in order to obtain usual $4 \times 4$ matrices, the numbers $1,2,3$, and 4 are assigned clockwise to the vertices of the rectangle).

$$
\left(\begin{array}{llll}
0 & a & b & c \\
a & 0 & c & b \\
b & c & 0 & a \\
c & b & a & 0
\end{array}\right)=a\left(\begin{array}{cccc}
0 & 1 & 0 & 0 \\
1 & 0 & 0 & 0 \\
0 & 0 & 0 & 1 \\
0 & 0 & 1 & 0
\end{array}\right)+b\left(\begin{array}{llll}
0 & 0 & 1 & 0 \\
0 & 0 & 0 & 1 \\
1 & 0 & 0 & 0 \\
0 & 1 & 0 & 0
\end{array}\right)+c\left(\begin{array}{llll}
0 & 0 & 0 & 1 \\
0 & 0 & 1 & 0 \\
0 & 1 & 0 & 0 \\
1 & 0 & 0 & 0
\end{array}\right) .
$$

The equivalence classes of the spaces $\left(X, d_{a}\right)$ will be called color components of $(X, d)$. Note that each $\left(X, d_{a}\right)$ is not exactly a metric space, because the "distances" $d_{a}$ can have 0 values outside the diagonal. But each such space corresponds to a unique equivalence class of a metric space, which can be obtained by replacing all nondiagonal 0 entries of $d_{a}$ with some positive number different from 1, bigger than 0.5 and smaller than 2. For instance, each color component of the rectangle is a square.

With these notations, the equivalence class of a metric space $(X, d)$ is given by its color components. The earlier remark about actions of groups on equivalence classes can be reformulated thus: A group $G$ acting on $X$ preserves the metric if and only if preserves the metric of all color components of $(X, d)$.

This has the following analogue.

Proposition 2.5. A coaction of $H$ on $X$ preserves the metric if and only if it preserves all its color components. 
Proof. Assume that $H$ preserves the metric and consider the multiplication and comultiplication of $\mathbb{C}(X)$ :

$$
m: \delta_{i} \otimes \delta_{j} \mapsto \delta_{i} \delta_{j}, \quad c: \delta_{i} \mapsto \delta_{i} \otimes \delta_{i}
$$

Then $m$ and $c$ intertwine $v^{\otimes 2}$ and $v$. It follows that their iterations

$$
m^{(k)}: \delta_{1} \otimes \cdots \otimes \delta_{k} \mapsto \delta_{1} \ldots \delta_{k}, \quad c^{(k)}: \delta_{i} \mapsto \delta_{i} \otimes \cdots \otimes \delta_{i}
$$

are in $\operatorname{Hom}\left(v^{\otimes k}, v\right)$ and $\operatorname{Hom}\left(v, v^{\otimes k}\right)$ respectively. In particular the operator

$$
d^{(k)}=m^{(k)} d^{\otimes k} c^{(k)}: \delta_{i} \mapsto \sum_{j} d(i, j)^{k} \delta_{j}
$$

is in the commutant End $v$ of $v$. Since $d^{(k)}$ is obtained from $d$ by raising all its entries to the power $k$, it has the decomposition

$$
d^{(k)}=\sum_{a \in C} a^{k} d_{a}
$$

If $A$ is the biggest color, the sequence $A^{-k} d^{(k)}$ of elements of End $v$ converges to $d_{A}$, so $d_{A}$ has to be an element of End $v$. By subtracting $\operatorname{Ad}_{A}$ from $d$ we get in this way by induction that each $d_{a}$ is in End $v$.

Corollary 2.6. Let $v: \mathbb{C}(X) \rightarrow \mathbb{C}(X) \otimes H$ be a metric-preserving coaction. If $K$ is an eigenspace of some color component $d_{a}$ then $v(K) \subset K \otimes H$.

Proof. Consider the orthogonal projection $P_{K}$ onto $K$. Since $K$ is an eigenspace of $d_{a}$ this projection is in the $\mathbb{C}^{*}$-algebra generated by $d_{a}$ in $\mathscr{L}(\mathbb{C}(X))$. Thus from $d_{a} \in$ End $v$ we get that $P_{K}$ is in End $v$ as well. On the other hand we have $v(K) \subset$ $K \otimes H$ if and only if $v$ commutes with $P_{K}$.

\section{Rules for computing the Hopf algebra}

In this section we find some rules for the computation of $H(X, d)$ and its corepresentations. There is a lot of structure on finite metric spaces that can be exploited. For instance simple geometric properties of $(X, d)$ lead to invariant subspaces or subalgebras of $v$ of dimension 1, 2 or 3 and the universal properties of the Hopf $\mathbb{C}^{*}$ algebras $\mathbb{C}^{*}(\mathbb{Z}), \mathbb{C}(\mathrm{SU}(2)), \mathbb{C}(\mathrm{SU}(2))_{-1}, \mathbb{C}\left(\mathbb{Z}_{2}\right)$ and $\mathbb{C}\left(\mathbb{Z}_{3}\right)$ can be used. However, the computation of $H(X, d)$ seems to be hard in the general case and the set of needed "rules" might be infinite. In fact we don't know what happens in the case $n=8$ and our less ambitious project here is just to find enough rules for dealing with the $n \leq 7$ case.

We begin with reformulations of three facts from the previous sections.

Lemma 3.1 (Triangle Rule). If $X$ has at most 3 points then $H(X, d)=\mathbb{C}(G(X, d))$. 
Lemma 3.2 (Simplex Rule). If $X$ is a simplex having at least 4 points the category of finite-dimensional corepresentations of $H(X, d)$ is the completion of the category of Temperley-Lieb diagrams of parameter

$$
\delta_{X}=\sqrt{\# X}
$$

Lemma 3.3 (Duplex Rule). If $X=Y \times Z$ is a product of simplices then the category of finite finite-dimensional corepresentations of $H(X, d)$ is the completion of the category of Fuss-Catalan diagrams of parameters

$$
\delta_{Y}=\sqrt{\# Y} \text { and } \delta_{Z}=\sqrt{\# Z} .
$$

Let $(X, d)$ be a finite metric space with color set $C \subset \mathbb{R}_{+}$. For any partition

$$
C=\left\{a_{1}, \ldots, a_{m}\right\} \cup\left\{b_{1}, \ldots, b_{n}\right\} \cup\left\{c_{1}, \ldots, c_{p}\right\} \cup \ldots
$$

we get a new space $\left(X^{\prime}, d^{\prime}\right)$ by identifying all $a$-colors, all $b$-colors, all $c$-colors etc. This is not exactly a metric space, but it corresponds to a unique equivalence class of a metric space (see comments on page 40 ). We say that $\left(X^{\prime}, d^{\prime}\right)$ is a decoloration of $(X, d)$.

As an example, consider the rectangle. Its color set has three elements $\{a, b, c\}$ so there are 5 possible partitions of it. The corresponding decolorations are the rectangle itself (no decoloration), the square (appearing 3 times, obtained by identifying 2 colors) and the 4-simplex (obtained by identifying all colors).

Each color component of $(X, d)$ being a decoloration of $(X, d)$, proposition 2.4 has the following slight generalisation plus reformulation.

Lemma 3.4 (Decoloration Rule). Let $\left(X^{\prime}, d^{\prime}\right)$ be a decoloration of $(X, d)$. Then $H(X, d)$ is a quotient of $H\left(X^{\prime}, d^{\prime}\right)$.

This can be used as follows. Let $(X, d)$ be a finite metric space and assume that we have found a decoloration $\left(X^{\prime}, d^{\prime}\right)$ such that $H\left(X^{\prime}, d^{\prime}\right)$ is commutative. Then $H(X, d)$ must be commutative as well, so it has is equal to $\mathbb{C}(G(X, d))$. A similar corollary holds with commutativity replaced by the transitivity notion discussed below.

We say that a color $a$ is cyclic if there exists a labelling of the points of $X$ such that the $a$ color connects 1 to 2 to 3 etc.

Lemma 3.5 (Cycle Rule). Assume there exists a cyclic color and that the space has $n \geq 5$ points. Then $H(X, d)=\mathbb{C}(G(X, d))$.

Proof. By using the decoloration rule it is enough to prove that if a Hopf $\mathbb{C}^{*}$-algebra $H$ coacts faithfully on the cycle $\left(X, d_{a}\right)$ then $H$ is commutative. 
The matrix of the cycle is

$$
M=\left(\begin{array}{cccccccc}
0 & 1 & 0 & 0 & \cdots & 0 & 0 & 1 \\
1 & 0 & 1 & 0 & \cdots & 0 & 0 & 0 \\
0 & 1 & 0 & 1 & \cdots & 0 & 0 & 0 \\
\vdots & \vdots & \vdots & \vdots & \ddots & \vdots & \vdots & \vdots \\
0 & 0 & 0 & 0 & \cdots & 1 & 0 & 1 \\
1 & 0 & 0 & 0 & \cdots & 0 & 1 & 0
\end{array}\right) .
$$

Let $w$ be a $n$-th root of 1 and consider the vector

$$
f=\left(\begin{array}{c}
1 \\
w \\
w^{2} \\
\cdots \\
w^{n-1}
\end{array}\right) .
$$

Then $f$ is an eigenvector of $M$ with eigenvalue $w+w^{n-1}$. Now by taking $w$ to be a primitive $n$-th root of 1 , it follows that all vectors $1, f, f^{2}, \ldots, f^{n-1}$ are eigenvectors of $M$. The invariant subspaces of $M$ are

$$
\mathbb{C} 1, \quad \mathbb{C} f \oplus \mathbb{C} f^{n-1}, \quad \mathbb{C} f^{2} \oplus \mathbb{C} f^{n-2}, \ldots,
$$

where the last subspace has dimension 1 or 2 depending on the parity of $n$. Write

$$
v(f)=f \otimes a+f^{n-1} \otimes b
$$

with $a, b \in H$ (see Corollary 2.6, to be used many times in what follows). By taking the square of this equality we get

$$
v\left(f^{2}\right)=f^{2} \otimes a^{2}+f^{n-2} \otimes b^{2}+1 \otimes(a b+b a) .
$$

It follows that $a b=-b a$ and that $v\left(f^{2}\right)$ is given by the formula

$$
v\left(f^{2}\right)=f^{2} \otimes a^{2}+f^{n-2} \otimes b^{2} .
$$

By multiplying this with $v(f)$ we get

$$
v\left(f^{3}\right)=f^{3} \otimes a^{3}+f^{n-3} \otimes b^{3}+f^{n-1} \otimes a b^{2}+f \otimes b a^{2} .
$$

But $n \geq 5$ implies that 1 and $n-1$ are different from 3 and from $n-3$, so we must have $a b^{2}=b a^{2}=0$. Now by using this and $a b=-b a$ we get by induction on $k$ that

$$
v\left(f^{k}\right)=f^{k} \otimes a^{k}+f^{n-k} \otimes b^{k}
$$

for any $k$. In particular for $k=n-1$ we get

$$
v\left(f^{n-1}\right)=f^{n-1} \otimes a^{n-1}+f \otimes b^{n-1} .
$$


On the other hand we have $f^{*}=f^{n-1}$, so by applying $*$ to $v(f)$ we get

$$
v\left(f^{n-1}\right)=f^{n-1} \otimes a^{*}+f \otimes b^{*} .
$$

Thus $a^{*}=a^{n-1}$ and $b^{*}=b^{n-1}$. Together with $a b^{2}=0$ this gives

$$
(a b)(a b)^{*}=a b b^{*} a^{*}=a b^{n} a^{n-1}=\left(a b^{2}\right) b^{n-2} a^{n-1}=0
$$

and from positivity we get $a b=0$. Together with $a b=-b a$ this shows that $a$ and $b$ commute. But $H$ is generated by the coefficients of $v$, which are powers of $a$ and $b$, so $H$ must be commutative.

Assume that the metric space $(X, d)$ has an even number of points. A star in $X$ is given by two colors $a$ and $b$ and a labelling of points of $X$ from 1 to $2 k$ such that $\left(X, d_{a}\right)$ consists of the segments $(1,2),(3,4), \ldots,(2 k-1,2 k)$ and $\left(X, d_{b}\right)$ consists of the segments $(2,3),(4,5), \ldots,(2 k-2,2 k-1)$. (If points of $X$ are arranged clockwise this doesn't quite look like a star; but a suitable rearrangement of points justifies terminology.)

Lemma 3.6 (Star Rule). If $X$ has a star then $H(X, d)=\mathbb{C}(G(X, d))$.

Proof. By using the projection $p_{\text {com }}$ it is enough to prove that $H(X, d)$ is commutative.

Let $\left(X^{\prime}, d^{\prime}\right)$ be the decoloration of $(X, d)$ obtained by identifying the colors $a$ and $b$. The star in $X$ becomes a cycle in $X^{\prime}$. From the decoloration rule we get that $H(X, d)$ is a quotient of $H\left(X^{\prime}, d^{\prime}\right)$, so if $H\left(X^{\prime}, d^{\prime}\right)$ is commutative then $H(X, d)$ is commutative as well. This is the case for $k \geq 3$, because we have $2 k \geq 6$ and the cycle rule applies.

In the remaining $k=2$ case $X$ must be a rectangle. Consider the vectors

$$
1=\left(\begin{array}{l}
1 \\
1 \\
1 \\
1
\end{array}\right), \quad A=\left(\begin{array}{r}
1 \\
-1 \\
1 \\
-1
\end{array}\right), \quad B=\left(\begin{array}{r}
1 \\
1 \\
-1 \\
-1
\end{array}\right), \quad C=\left(\begin{array}{r}
1 \\
-1 \\
-1 \\
1
\end{array}\right) .
$$

By using the color decomposition of the rectangle given in Section 3 we see that these are eigenvectors for all three matrix distances, corresponding to different eigenvalues. By Corollary 2.6 the three 1-dimensional spaces spanned by $A, B$ and $C$ must be left invariant by the coaction, so we must have

$$
v(A)=A \otimes a, \quad v(B)=B \otimes b, \quad v(C)=C \otimes c
$$

for some $a, b, c \in H$. We have between $A, B, C$ the relations

$$
A B=B A=C, \quad A C=C A=B, \quad C B=B C=A,
$$


and by applying $v$ we get from the multiplicativity of $v$ that $a, b, c$ satisfy similar formulae, hence commute. But $a, b, c$ generate $H$, so $H$ is commutative.

A coaction $v: \mathbb{C}(X) \rightarrow \mathbb{C}(X) \otimes H$ is called transitive if $v(f)=f \otimes 1$ implies that $f$ is a constant function. This is an extension of the classical notion of transitivity: in the case $H=\mathbb{C}(G)$ we have the formulae

$$
v(f)=\sum_{j} \delta_{j} \otimes \sum_{g} f\left(g^{-1} j\right) \delta_{g}, \quad f \otimes 1=\sum_{j} \delta_{j} \otimes \sum_{g} f(j) \delta_{g},
$$

so the coaction of $\mathbb{C}(G)$ is transitive if and only if the action of $G$ has one orbit.

A metric space $(X, d)$ is called homogeneous if its automorphism group acts transitively, meaning has one orbit. This is the same as asking that for any $x, y \in X$ there is a metric-preserving permutation $g \in \mathscr{Y}_{X}$ such that $g x=y$.

One can say that $(X, d)$ is "quantum homogeneous" if the coaction of $H(X, d)$ is transitive in the above sense. We have here the following necessary condition.

Lemma 3.7 (Magic Rule). If the coaction of $H(X, d)$ is transitive then the following condition must be satisfied. For any two points $x, y \in X$ there exists a bijection $\sigma: X \rightarrow X$ such that $\sigma(x)=y$ and such that $d(x, z)=d(y, \sigma(z))$ for any $z$.

The terminology comes from the following fact. Assume that $X$ has $n$ points and that there are exactly $n-1$ colors. By replacing these colors with the numbers from 1 to $n-1$, the matrix distance must be a magic matrix, i.e., a $n \times n$ matrix having the numbers from 0 to $n-1$ on each row and column. As an example, here is the magic matrix corresponding to the rectangle.

$$
\left(\begin{array}{llll}
0 & 1 & 2 & 3 \\
1 & 0 & 3 & 2 \\
2 & 3 & 0 & 1 \\
3 & 2 & 1 & 0
\end{array}\right) .
$$

Proof. For any color $a$ we have $d_{a} \in$ End $v$, so $d_{a}(1)$ is fixed by the coaction. But $d_{a}(1)$ is scalar if and only if the matrix $d_{a}$ has the same number of 1's on each row.

We say that a color $a$ is bicyclic if $\left(X, d_{a}\right)$ consists of two disjoint cycles $C_{n} \sqcup C_{N}$.

Lemma 3.8 (Bicycle Rule). Assume there exists a bicyclic color component of the form $C_{n} \sqcup C_{N}$ with $n \neq N$. Then the coaction of $H(X, d)$ is not transitive.

Proof. By using the decoloration rule it is enough to prove that if a Hopf $\mathbb{C}^{*}$-algebra $H$ coacts on the bicycle then the coaction is not transitive.

If one of $n$ and $N$ is 2 then the magic rule shows that $H(X, d)$ is not transitive. So we may assume $n, N \geq 3$. Label the points of $X$ in such a way that the matrix 
of distances is

$$
M=\left(\begin{array}{cc}
M_{n} & 0 \\
0 & M_{N}
\end{array}\right)
$$

where $M_{n}$ and $M_{N}$ are the matrices of the two cycles. Let $w$ be a primitive $n$-th root of unity and consider the vector $f$ consisting of powers of $w$. Then the eigenvalues and eigenvectors of the matrix $\left(\begin{array}{cc}M_{n} & 0 \\ 0 & 0\end{array}\right)$ are given by

$$
\begin{aligned}
2: & {\left[\left(\begin{array}{l}
1 \\
0
\end{array}\right)\right], } \\
\left(w+w^{n-1}\right): & {\left[\left(\begin{array}{l}
f \\
0
\end{array}\right),\left(\begin{array}{c}
f^{n-1} \\
0
\end{array}\right)\right], } \\
\left(w^{2}+w^{n-2}\right): & {\left[\left(\begin{array}{c}
f^{2} \\
0
\end{array}\right),\left(\begin{array}{c}
f^{n-2} \\
0
\end{array}\right)\right] \ldots }
\end{aligned}
$$

where each eigenvalue is followed by its eigenvectors and where the end of the sequence depends on the parity of $n$. Similarly, the matrix $\left(\begin{array}{ll}0 & 0 \\ 0 & M_{N}\end{array}\right)$ gives

$$
\begin{aligned}
2: & {\left[\left(\begin{array}{l}
0 \\
1
\end{array}\right)\right], } \\
\left(W+W^{N-1}\right): & {\left[\left(\begin{array}{c}
0 \\
F
\end{array}\right),\left(\begin{array}{c}
0 \\
F^{N-1}
\end{array}\right)\right], } \\
\left(W^{2}+W^{N-2}\right): & {\left[\left(\begin{array}{c}
0 \\
F^{2}
\end{array}\right),\left(\begin{array}{c}
0 \\
F^{N-2}
\end{array}\right)\right] \ldots }
\end{aligned}
$$

where $W$ is a primitive $N$-th root of unity and $F$ is the vector consisting of powers of $W$. Since $n \neq N$ it follows that the decomposition corresponding to $M$ is

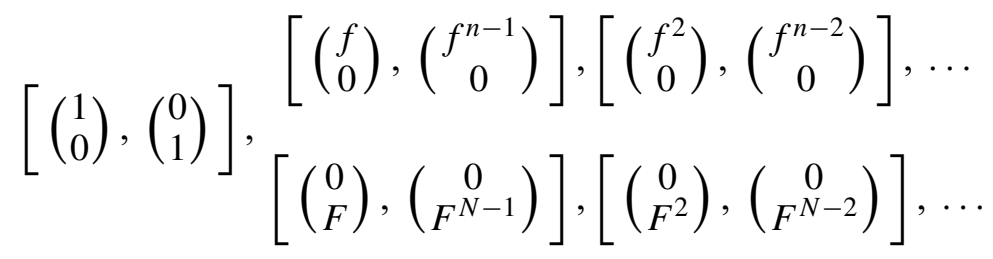

where the two series might interfere on their last entries, depending on the parity of $n$ and $N$. Consider the first subspace, corresponding to the eigenvalue 2 :

$$
\left[\left(\begin{array}{l}
1 \\
0
\end{array}\right),\left(\begin{array}{l}
0 \\
1
\end{array}\right)\right]=\left[\left(\begin{array}{l}
1 \\
1
\end{array}\right),\left(\begin{array}{r}
1 \\
-1
\end{array}\right)\right] .
$$

By Corollary 2.6 this space is invariant by $v$, so we can find $\xi, u \in H$ such that

$$
v\left(\begin{array}{r}
1 \\
-1
\end{array}\right)=\left(\begin{array}{l}
1 \\
1
\end{array}\right) \otimes \xi+\left(\begin{array}{r}
1 \\
-1
\end{array}\right) \otimes u .
$$


From the trace-preservation property of $v$ we get that $\operatorname{tr}\left(\begin{array}{r}1 \\ -1\end{array}\right) 1=0$ is equal to

$$
(\operatorname{tr} \otimes \mathrm{id}) v\left(\begin{array}{r}
1 \\
-1
\end{array}\right)=\operatorname{tr}\left(\begin{array}{l}
1 \\
1
\end{array}\right) \xi+\operatorname{tr}\left(\begin{array}{r}
1 \\
-1
\end{array}\right) u=2 \xi .
$$

It follows that $\xi=0$, so we have

$$
v\left(\begin{array}{r}
1 \\
-1
\end{array}\right)=\left(\begin{array}{r}
1 \\
-1
\end{array}\right) \otimes u .
$$

From this we get that $v$ is given on the subspace by

$$
\begin{aligned}
& v\left(\begin{array}{l}
1 \\
0
\end{array}\right)=\left(\begin{array}{l}
1 \\
0
\end{array}\right) \otimes \frac{1+u}{2}+\left(\begin{array}{l}
0 \\
1
\end{array}\right) \otimes \frac{1-u}{2} \\
& v\left(\begin{array}{l}
0 \\
1
\end{array}\right)=\left(\begin{array}{l}
1 \\
0
\end{array}\right) \otimes \frac{1-u}{2}+\left(\begin{array}{l}
0 \\
1
\end{array}\right) \otimes \frac{1+u}{2} .
\end{aligned}
$$

Assume that one of $n$ and $N$ is greater than 5, say $n \geq 5$. By arguing like in the proof of the cycle rule we get from a formula of type

$$
v\left(\begin{array}{l}
f \\
0
\end{array}\right)=\left(\begin{array}{l}
f \\
0
\end{array}\right) \otimes a+\left(\begin{array}{c}
f^{n-1} \\
0
\end{array}\right) \otimes b
$$

that the following formula is true for any $k$.

$$
v\left(\begin{array}{c}
f^{k} \\
0
\end{array}\right)=\left(\begin{array}{c}
f^{k} \\
0
\end{array}\right) \otimes a^{k}+\left(\begin{array}{c}
f^{n-k} \\
0
\end{array}\right) \otimes b^{k} .
$$

In particular for $k=n$ we get

$$
v\left(\begin{array}{l}
1 \\
0
\end{array}\right)=\left(\begin{array}{l}
1 \\
0
\end{array}\right) \otimes a^{n}+\left(\begin{array}{l}
1 \\
0
\end{array}\right) \otimes b^{n}=\left(\begin{array}{l}
1 \\
0
\end{array}\right) \otimes\left(a^{n}+b^{n}\right)
$$

and comparison with the above formula for $v\left(\begin{array}{l}1 \\ 0\end{array}\right)$ gives $u=1$. It follows that $\left(\begin{array}{c}1 \\ -1\end{array}\right)$ is a fixed vector of the coaction, so the coaction is not transitive.

The remaining case is $n, N \leq 4$. Since $n, N$ are different and both are greater than 3 , one is 3 and the other is 4 . Assume $N=4$ and consider the vector

$$
\gamma=F^{2}=\left(\begin{array}{r}
1 \\
-1 \\
1 \\
-1
\end{array}\right) \text {. }
$$

This is the unique eigenvector of $M_{N}$ corresponding to the eigenvalue 0 . Since $n=3$ is odd, $M_{n}$ has no eigenvalue 0 . It follows that $\left(\begin{array}{l}0 \\ \gamma\end{array}\right)$ is the unique eigenvector 
of $M$ corresponding to the eigenvalue 0 , so we must have

$$
v\left(\begin{array}{l}
0 \\
\gamma
\end{array}\right)=\left(\begin{array}{l}
0 \\
\gamma
\end{array}\right) \otimes c
$$

for some $c \in H$. By taking the square of this equality we get

$$
v\left(\begin{array}{l}
0 \\
1
\end{array}\right)=\left(\begin{array}{l}
0 \\
1
\end{array}\right) \otimes c^{2}
$$

and comparison with the above formula for $v\left(\begin{array}{l}1 \\ 0\end{array}\right)$ gives $u=1$. Once again, it follows that $\left(\begin{array}{r}1 \\ -1\end{array}\right)$ is a fixed vector of the coaction, so the coaction is not transitive.

\section{Classification for $n \leq 7$}

The purpose of this section is to compute the corepresentation theory invariants of the Hopf algebra $H(X, d)$, in case the metric space $(X, d)$ has at most 7 points.

In order to cut off unwanted complexity, there is a natural condition to be put on $(X, d)$, namely quantum homogeneity. Recall from previous section that $(X, d)$ is said to be quantum homogeneous when the universal coaction $v: \mathbb{C}(X) \rightarrow \mathbb{C}(X) \otimes$ $H(X, d)$ is transitive, meaning that $v(f)=f \otimes 1$ implies that $f$ is a scalar function.

The motivating remark here is that functions satisfying $v(f)=f \otimes 1$ are those belonging to the relative commutant of the associated subfactor [Banica 2002a], so quantum homogeneity corresponds to the well-known irreducibility condition in subfactors.

It is clear from definitions that a homogeneous space is quantum homogeneous. It turns out that for spaces having at most 7 points the converse holds. We do not know if these notions are equivalent in general.

The decoloration rule shows that all color components of a quantum homogeneous space must be quantum homogeneous. These color components are graphs, so our first task is to prove that for a graph having at most 7 vertices, homogeneity is equivalent to quantum homogeneity.

The complement of a graph $(X, d)$ with vertex set $X$ and incidence matrix $d$ is by definition the graph $(X, \mathbb{1}-1-d)$, where $\mathbb{1}$ is the matrix filled with 1 's. Since $v \mathbb{1}=\mathbb{1} v=\mathbb{1}$ for any magic biunitary matrix $v$, we have $H(X, d)=H(X, \mathbb{1}-1-d)$.

Lemma 4.1. A graph with $n \leq 7$ vertices is homogeneous if and only if it is quantum homogeneous. There are 24 such graphs:

- 1 point.

- 2 points, segment.

- 3 points, triangle.

- 4 points, tetrahedron, 2 segments, square. 
- 5 points, 5-simplex, 5-cycle.

- 6 points, 6-simplex, plus 6-cycle, 3 segments, 2 triangles, and their complements.

- 7 points, 7-simplex, 7-cycle and its complement.

Proof. Homogeneous implies quantum homogeneous, and all graphs in the list are homogeneous. It remains to prove that any quantum homogeneous graph with $n \leq 7$ vertices is in the list.

Let $(X, d)$ be such a graph. The magic rule applies and shows that the incidence matrix $d$ has the same number of 1's, say $k$, on each of its rows and columns. Thus $(X, d)$ must be a $k$-regular graph, for a certain number $k$. By replacing the graph with its complement we may assume that that the valence $k$ of the graph is smaller or equal than the valence $n-k-1$ of its complement.

Summing up, it remains to prove the following assertion. Any quantum homogeneous $k$-regular graph with $n$ vertices, $2 k+1 \leq n \leq 7$, is in the list.

For $k=0$ the graph must be $n$ points and no edges, in the list.

For $k=1$ the only graphs are the 2 or 3 segments, in the list.

For $k=2$ the graph must be a union of $m$-cycles with $m>3$. The bicycle rule shows that $m$ must be the same for all of these $m$-cycles, and the graphs left are the $n$-cycles with $3 \leq n \leq 7$, plus the 2 triangles, all of them in the list.

For $k \geq 3$ we must have $n \geq 2 \times 3+1=7$. The only possible case is $k=3$ and $n=7$, but this is excluded by the observation that the incidence matrix must have $n k / 2$ values of 1 above the diagonal.

Theorem 4.2. Let $(X, d)$ be a metric space having at most 7 points. Then one of the following happens.

(1) The coaction of $H(X, d)$ is not transitive.

(2) The coaction of $H(X, d)$ is not transitive. $H(X, d)$ is the algebra of functions on the usual automorphism group of $(X, d)$.

(3) The coaction of $H(X, d)$ is not transitive. $X$ is a simplex and the category of finite-dimensional corepresentations of $H(X, d)$ is the completion of the category of Temperley-Lieb diagrams of parameter $\delta_{X}=\sqrt{\# X}$.

(4) The coaction of $H(X, d)$ is not transitive. $X=Y \times Z$ is a product of simplices and the category of finite-dimensional corepresentations of $H(X, d)$ is the completion of the category of Fuss-Catalan diagrams of parameters $\delta_{Y}=$ $\sqrt{\# Y}$ and $\delta_{Z}=\sqrt{\# Z}$.

Proof. If $X$ is a simplex the triangle or simplex rule applies and shows that we are in situation (2) or (3). If $X$ doesn't satisfy the magic rule we are in situation (1). Thus it remains to prove the following statement. 
Let $(X, d)$ be metric space having $n=4,5,6$ or 7 points, having at least two colors and satisfying the magic rule. Then (1), (2) or (4) holds.

For $n=4$ the only spaces left are the square and the rectangle. The duplex rule applies to the square and the star rule applies to the rectangle.

For $n=5$ or 7 Lemma 4.1 shows that all color components must be $n$-cycles. The cycle rule applies and shows that we are in situation (2).

For $n=6$ Lemma 4.1 shows that all color components must be among the following 6 graphs: 6 -cycle, 3 segments, 2 triangles, and their complements. If there is a 6-cycle the cycle rule applies and (2) must hold. The same happens whenever the complement of the 6-cycle is present. In the remaining cases, either the duplex rule applies and (4) must hold, or the star rule applies and we are in situation (2).

In the $n=8$ case the situation is a bit more complicated. There are 14 homogeneous graphs with 8 vertices, and an analogue of Lemma 4.1 holds. Techniques in this paper apply to most of them, and there are 3 graphs left. First is the cube, which can be shown to correspond to a tensor product between 2 Temperley-Lieb algebras. Second is a graph which looks like a wheel with 8 spokes, which gives $\mathbb{C}\left(D_{8}\right)$. Third is the graph formed by 2 squares. This corresponds to a Fuss-Catalan algebra on 3 colors, but the proof is quite long.

As for the passage from graphs to metric spaces, or colored graphs, this is done by an easy application of the decoloration rule, as in proof of Theorem 4.2. However, there is a problem at $n=8$, with one space left, which is formed by 2 rectangles. We don't know yet how to compute invariants of the associated Hopf algebra.

For $n=9$ an analogue of Lemma 4.1 holds once again, but the proof is long. All graphs give $\mathbb{C}\left(D_{9}\right)$ or correspond to Fuss-Catalan algebras, except for an exceptional graph, which looks like a torus. So far, we have no results about this graph.

\section{References}

[Banica 2000] T. Banica, "Compact Kac algebras and commuting squares”, J. Funct. Anal. 176:1 (2000), 80-99. MR 2003a:46086 Zbl 0969.46048

[Banica 2002a] T. Banica, “The planar algebra of a coaction”, 2002. math.QA/0207035

[Banica 2002b] T. Banica, "Quantum groups and Fuss-Catalan algebras", Comm. Math. Phys. 226:1 (2002), 221-232. MR 2002k:46178 Zbl 1034.46062

[Bhattacharyya 2002] B. Bhattacharyya, "A note on intermediate subfactors of the Krishnan-Sunder subfactors", 2002. math.OA/0211015

[Bisch and Jones 1997] D. Bisch and V. Jones, "Algebras associated to intermediate subfactors", Invent. Math. 128:1 (1997), 89-157. MR 99c:46072 Zbl 0891.46035

[Bisch and Jones 2000] D. Bisch and V. Jones, "Singly generated planar algebras of small dimension”, Duke Math. J. 101:1 (2000), 41-75. MR 2002f:46118 Zbl 01425270 
[Bisch and Jones 2003] D. Bisch and V. Jones, "Singly generated planar algebras of small dimension, II", Adv. Math. 175:2 (2003), 297-318. MR 2004d:46073 Zbl 1041.46048

[Connes 1996] A. Connes, "Gravity coupled with matter and the foundation of non-commutative geometry”, Comm. Math. Phys. 182:1 (1996), 155-176. MR 98f:58024 Zbl 0881.58009

[Jones 1985] V. F. R. Jones, “A polynomial invariant for knots via von Neumann algebras", Bull. Amer. Math. Soc. (N.S.) 12:1 (1985), 103-111. MR 86e:57006 Zbl 0564.57006

[Jones 2000] V. F. R. Jones, "The planar algebra of a bipartite graph”, pp. 94-117 in Knots in Hellas '98 (Delphi, 1998), edited by C. M. Gordon et al., Ser. Knots Everything 24, World Sci. Publishing, River Edge (NJ), 2000. MR 2003c:57003 Zbl 1021.46047

[Jones and Sunder 1997] V. Jones and V. S. Sunder, Introduction to subfactors, London Mathematical Society Lecture Note Series 234, Cambridge University Press, Cambridge, 1997. MR 98h:46067 Zbl 0903.46062

[Kauffman 1987] L. H. Kauffman, "State models and the Jones polynomial”, Topology 26:3 (1987), 395-407. MR 88f:57006 Zbl 0622.57004

[Landau 2002] Z. A. Landau, "Exchange relation planar algebras", Geom. Dedicata 95 (2002), 183214. MR 2003k:46091 Zbl 1022.46039

[Landau and Sunder 2002] Z. Landau and V. S. Sunder, "Planar depth and planar subalgebras", $J$. Funct. Anal. 195:1 (2002), 71-88. MR 2003j:46096 Zbl 1030.46078

[Paschke and Sitarz 1998] M. Paschke and A. Sitarz, "Discrete spectral triples and their symmetries”, J. Math. Phys. 39:11 (1998), 6191-6205. MR 99k:58017 Zbl 0934.58006

[Rieffel 2004] M. A. Rieffel, Gromov-Hausdorff distance for quantum metric spaces, Mem. Amer. Math. Soc. 168, Amer. Math. Soc., Providence, 2004. MR 2055926 math.OA/0011063

[Wang 1995] S. Wang, "Free products of compact quantum groups", Comm. Math. Phys. 167:3 (1995), 671-692. MR 95k:46104 Zbl 0838.46057

[Wang 1998] S. Wang, "Quantum symmetry groups of finite spaces", Comm. Math. Phys. 195:1 (1998), 195-211. MR 99h:58014 Zbl 1013.17008

[Woronowicz 1987] S. L. Woronowicz, "Compact matrix pseudogroups", Comm. Math. Phys. 111:4 (1987), 613-665. MR 88m:46079 Zbl 0627.58034

[Woronowicz 1988] S. L. Woronowicz, "Tannaka-Kreĭn duality for compact matrix pseudogroups: Twisted SU(N) groups”, Invent. Math. 93:1 (1988), 35-76. MR 90e:22033 Zbl 0664.58044

[Woronowicz 1998] S. L. Woronowicz, "Compact quantum groups", pp. 845-884 in Symétries quantiques = Quantum symmetries (Les Houches, 1995), edited by A. Connes et al., Elsevier, Amsterdam, 1998. MR 99m:46164 Zbl 0997.46045

Received April 30, 2003. Revised February 10, 2004.

\author{
TEODOR BANICA \\ DePARTEMENT DE MATHÉMATIQUeS \\ UNIVERSITÉ PAUL SABATIER \\ 118 ROUTE DE NARBONNE \\ 31062 TOULOUSE \\ FRANCE \\ banica@picard.ups-tlse.fr
}

